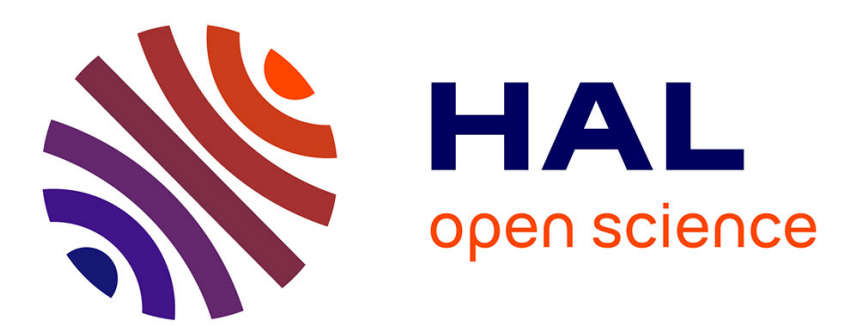

\title{
Sensitivity of train stochastic dynamics to long-time evolution of track irregularities
}

Nicolas Lestoille, Christian Soize, Christine Funfschilling

\section{To cite this version:}

Nicolas Lestoille, Christian Soize, Christine Funfschilling. Sensitivity of train stochastic dynamics to long-time evolution of track irregularities. Vehicle System Dynamics, 2016, 54 (5), pp. 545-567. 10.1080/00423114.2016.1142095 . hal-01276447

\section{HAL Id: hal-01276447 \\ https://hal.science/hal-01276447}

Submitted on 19 Feb 2016

HAL is a multi-disciplinary open access archive for the deposit and dissemination of scientific research documents, whether they are published or not. The documents may come from teaching and research institutions in France or abroad, or from public or private research centers.
L'archive ouverte pluridisciplinaire HAL, est destinée au dépôt et à la diffusion de documents scientifiques de niveau recherche, publiés ou non, émanant des établissements d'enseignement et de recherche français ou étrangers, des laboratoires publics ou privés. 


\title{
Sensitivity of train stochastic dynamics to long-time evolution of track irregularities
}

\author{
N. Lestoille $\mathrm{a}^{\mathrm{a}, \mathrm{b}}$, C. Soize $\mathrm{S}^{\mathrm{a} *}$ and C. Funfschilling ${ }^{\mathrm{b}}$ \\ a Université Paris Est, Laboratoire Modélisation et Simulation Multi-Echelle, MSME \\ UMR 8208 CNRS, 5 bd Descartes, 77454 Marne-la-Vallée, France; b SNCF, Innovation \\ and Research Department, Immeuble Lumière, 40 av des Terroirs de France, 75611 Paris \\ Cedex 12, France
}

(February 2015)

\begin{abstract}
The influence of the track geometry on the dynamic response of the train is of great concern for the railway companies, because they have to guarantee the safety of the train passengers in ensuring the stability of the train. In this paper, the long-term evolution of the dynamic response of the train on a stretch of the railway track is studied with respect to the long-term evolution of the track geometry. The characterization of the long-term evolution of the train response allows the railway companies to start off maintenance operations of the track at the best moment. The study is performed using measurements of the track geometry, which are carried out very regularly by a measuring train. A stochastic model of the studied stretch of track is created in order to take into account the measurement uncertainties in the track geometry. The dynamic response of the train is simulated with a multibody software. A noise is added in output of the simulation to consider the uncertainties in the computational model of the train dynamics. Indicators on the dynamic response of the train are defined, allowing to visualize the long-term evolution of the stability and the comfort of the train, when the track geometry deteriorates.
\end{abstract}

Keywords: train dynamics; stochastic modeling; track irregularities; high-speed trains

\section{Introduction}

The tracks for the high speed trains are submitted to more and more solicitations, because of the increase of the train traffic, the load and the speed of the trains. These solicitations induce degradations of the track geometry, making evolve the track irregularities. Such degradations impact the dynamic response of the train in return. To guarantee a good level of safety and comfort of the train, maintenance operations of the track have to be regularly undertaken. These maintenance operations are heavy and costly. They would gain being started off by indicators on the train safety and comfort, and no more only on the track-irregularities measurements. However, the vehicle-track system is highly nonlinear, and there is no obvious correlation between track irregularities and the vehicle-track forces (see [1]). The knowledge of the influence of the track geometry on the dynamic response of the train needs to be increased in order to define indicators for the simulated dynamic response of the train, and to characterize their evolution. Taking into account parameters uncertainties and model uncertainties increases the robustness of indicators.

*Corresponding author. Email: christian.soize@univ-paris-est.fr 
In Section 2, the adopted approach is detailed. Then, Section 3 focuses on the track geometry modeling, taking into acount the measurements for a given stretch of track. In Section 4, indicators on the train dynamics are defined to assess the train behavior on this given stretch. Model uncertainties are identified in order to have dynamic indicators more robust. The sensitivity of the indicators to time evolution of the track irregularities is exposed in Section 5.

\section{Proposed approach}

The goal of this work is to set up robust indicators, able to describe the longterm evolution of the dynamic response of the train, under the influence of the evolution of the track irregularities. The distinction has to be done between the long-term evolution (of the order of the month), which will be denoted by $\tau$, and the characteristic time of the train dynamics (of the order of the second), which is denoted by $t$. Actually, the vehicle-track system is a complex system, with high nonlinearities and coupling between inputs (track geometry, track stiffness, train mechanical characteristics) and outputs (train responses). The track geometry is the main source of excitation for the train. In France, SNCF measures the track geometry very precisely and frequently, which provides us information on the track geometry in the long time $\tau$. A global stochastic model of the track geometry has been built by Perrin et al. in [2] using a very large experimental data basis concerning a complete high-speed line. This stochastic modeling is very useful to carry out nonlinear stochastic dynamic analysis of the train excited by the random track geometry. We need now to adapt this global stochastic model for a given stretch of track, in order to observe its long-term evolution, by constructing a local stochastic model. The adaptation of the global stochastic model to a local stochastic model is performed in Section 3.4, by introducing an additional stochastic model as an input noise in the global stochastic model proposed by Perrin et al.. The introduction of the input noise allows the measurement errors and the local variability to be taken into account in the modeling of the track irregularities, using the experimental measured data that are available for this track stretch.

Moreover, the analysis of the long-term evolution of the vehicle-track system was usually done regarding to the long-term evolution of the track geometry irregularities. We noticed in [3] that the high number of coefficients in the global stochastic model developed for representing track geometry irregularities makes it difficult to study its long-term evolution. Besides, the long-term evolution of the track geometry does not always give precise information about the long-term evolution of the dynamic response of the train because of the nonlinearity between the track geometry and the train response. This nonlinearity is due to the wheel-rail contact forces, to the train suspensions, and to bumpstops in the train suspensions. For example, a particular sequence of small track irregularities may cause a less stable reaction of the train than one big irregularity (see $[4,5])$. That is why the longterm evolution of the vehicle-track system is analyzed in this work focusing on the dynamic response of the train.

The dynamic response of the train is numerically simulated using the adapted stochastic model of the track geometry relative to the given track stretch. The inputs for the simulation are the track design, the track irregularities modeled with the adapted stochastic model, and a model of the train. For the numerical simulation, the computational model of the train is a multibody dynamic model. In this paper, the train considered is the French double-decker high-speed train TGV Duplex. The simulation outputs are accelerations in the train and wheel-rail contact forces. To assess the long-term evolution of the train dynamics and start 
off maintenance operations, we have to define robust indicators for the dynamic response of the train. Those indicators are computed using the simulation outputs and give information about the stability and the comfort of the train.

Moreover, in order to increase the robustness of the chosen dynamic indicators, model uncertainties have to be taken into account in the train modeling. Those uncertainties are estimated in Section 4.3 by comparing simulation outputs (responses of the train) with experimental measurements of the train response. The characterization of the model uncertainties consists in identifying a stochastic output noise that is added to the dynamic indicators.

- The goal of this paper is to analyze the sensitivity of indicators related to the train dynamic responses due to a long-term evolution of track irregularities that are modeled by a random field for a given stretch of track, and using experimental measurements of the track geometry and of the train dynamics. Taking into account the strong heterogeneity of the track and its substructure along a complete high-speed line, the description of the long-term evolution in terms of physical parameters would require several thousand parameters with an additional lack of knowledge concerning the mechanical models. Consequently, such an approach is considered, in the present state-of-the-art, not realistic for a long-term prediction, and a statistical model based on true measurements seems to be a way for obtaining a robust prediction model. Thus, the goal is not to analyze the long-term evolution of some physical parameters of the track and of its underlying structure, or the long-term evolution of some mechanical and geometrical parameters of the train and of the wheel-rail contact.

- This choice is guided by the fact that the railway network is spatially extremely heterogeneous for a same railway line. Moreover, the long-term evolution of the track depends on the considered stretch of the track and is related to the weather conditions and to the various types of trains that run on the line. Thus, a parametric approach is not feasible.

- This is why a global stochastic model of the track irregularities for the French railway network is constructed and adapted for all considered stretches of the track with measurements of these stretches, which experimentally validate the constructed stochastic model.

- Furthermore, the train is used as a tool to characterize the impacts of the track irregularities on the comfort and the security of the train. In these conditions, it is important to take into account the modeling error in the train computational model, which is identified using simultaneous experimental measurements of a track geometry and of the corresponding responses of the train. Such an approach replaces a parameterized model of the train dynamics and of the vehicle-track interaction, for which the construction is not feasible.

\section{Stochastic modeling of track irregularities using experimental measurements}

\section{1. $\quad$ Track measurements}

The track geometry is measured very precisely and very frequently by a measuring train equipped with laser cameras (see $[6,7]$ ). The track is described by two data sets:

- the initial track design, which corresponds to the theoretical track (as it was planned before the construction) and which is made of perfect lines and curves. 
- the irregularities of the track which appear during the track life cycle and which have to be added to the track design. We consider track irregularities along the track, for which the wavelengths are between 3 and $150 \mathrm{~m}$, and for which the order of magnitude of the amplitude is one millimeter to a dozen of millimeters. The track irregularities change over long time $\tau$.

The track irregularities are modeled by a vector-valued random field $\mathbf{Y}$, which depends on long-time parameter $\tau$, and which is denoted by

$$
s \mapsto \mathbf{Y}(s ; \tau)=\left(Y_{1}(s ; \tau), Y_{2}(s ; \tau), Y_{3}(s ; \tau), Y_{4}(s ; \tau)\right)
$$

This random field is indexed by the curvilinear abscissa along the track stretch $s$ in $\Omega=[0, S]$, where $S$ is the fixed length of a stretch of track. Long time $\tau$ is a discrete parameter that increases between successive measurements of the given track stretch,

$$
\tau_{0}<\tau_{1}<\tau_{2}<\ldots<\tau_{K}
$$

in which $\tau_{0}$ is the time of the first measurement performed just after a maintenance operation, and where $\tau_{1}, \tau_{2}, \ldots, \tau_{K}$ correspond to the successive long times for which there are measurements of the track geometry, and $\tau_{K}$ is the time of the last measurement before the next maintenance operation.

\subsection{A few comments about existing modelings of track irregularities}

In order to characterize and to generate track irregularities, various modeling methods for the track irregularities have been previously developed. First models are deterministic and use spectral representations or wavelet transforms of the measured track irregularities. Because these models are not able to take into account the variability of the track geometry, more advanced modelings have recently been developed, which consist in stochastic models whose statistical properties are identified by using statistical inverse methods with experimental measurements of the track irregularities. Another approach is proposed in [8] by introducing a representation of the track irregularities based on a discrete Fourier transform. A model based on the use of the classical spectral representation for stationary Gaussian or non-Gaussian random processes [9, 10], have been proposed. These representations allow for generating track irregularities in order to compute the dynamic response of the train with respect to the track irregularities. The statistical dependencies between the track irregularities, highlighted in [11], recommends to consider the four track irregularities simultaneously.

More recently, the Perrin works $[2,4,5,12]$ consider the four track irregularities as a non-stationary and non-Gaussian vector-valued random field, whose spatial discretization is a non-Gaussian random vector. This global non-stationary nonGaussian stochastic model as been constructed and identified using a big data basis of experimental measurements of the track geometry for each high-speed lines of the French railway network. Nevertheless, this global stochastic model is not sufficiently sensitive to the local degradation of the track irregularities of a given local track stretch, because they do not preserve the specificity of each given track stretch. This is the reason why, in this work, a local model is proposed (see Section 3.4) by adapting this global stochastic model. 


\subsection{Global stochastic modeling of the track irregularities}

A global stochastic model of the track irregularities has been proposed in [2] and detailed in [4, 13-16]. This model has been built solving an inverse statistical problem using a very large experimental data base related to a complete high-speed line of the French railway network. It is very robust with respect to measurement errors and has the capability to generate the track irregularities for any given stretch belonging to this high-speed line.

The track irregularities vector $\mathbf{Y}=\left(Y_{1}, Y_{2}, Y_{3}, Y_{4}\right)$, introduced in Equation (1), is modeled by a vector-valued random field, defined on a probability space $(\Theta, \mathcal{F}, \mathcal{P})$, indexed by $\Omega=[0, S]$, with values in $\mathbb{R}^{4}$. In the global stochastic model, $\mathbf{Y}$ is supposed to be independent of long time $\tau$. Then, for one fixed $s$ in $\Omega$, the measurements $\left\{Y\left(s, \tau_{0}\right), \ldots, Y\left(s, \tau_{K}\right)\right\}$ are considered as independent realizations of the random vector $\mathbf{Y}(s)$. It has been proved that random field $\mathbf{Y}$ is neither Gaussian nor homogeneous (not stationary). Random field $\mathbf{Y}$ is centered,

$$
E\{\mathbf{Y}(s)\}=0 \quad, \quad \forall s \in[0, S]
$$

where $E\{$.$\} is the mathematical expectation. The continuous vector-valued ran-$ dom field $\mathbf{Y}(s), s \in \Omega$, is replaced by its spatial discretization at curvilinear abscissa $s_{n}=n h$, with $h$ the measurement spatial step, $n=0, \ldots, N_{s}$, and $N_{s}=S / h$. Keeping the same notation for the continuous random field and its spatial discretization, the following random vector $\mathbf{X}=\left(\mathbf{X}^{1}, \mathbf{X}^{2}, \mathbf{X}^{3}, \mathbf{X}^{4}\right)$ with values in $\mathbb{R}^{4\left(N_{s}+1\right)}$, is introduced such that

$$
\mathbf{X}^{k}=\left(Y_{k}(0), Y_{k}(h), Y_{k}(2 h), \ldots, Y_{k}\left(N_{s} h\right)\right) \quad, \quad k=1,2,3,4,
$$

with values in $\mathbb{R}^{N_{s}+1}$. In the global stochastic model, the centered random vector $\mathbf{X}$ is written using a truncated principal component decomposition such as

$$
\mathbf{X} \simeq \sum_{i=1}^{N_{\eta}} \sqrt{\lambda_{i}} \mathbf{u}_{i} \eta_{i},
$$

in which $N_{\eta} \ll 4\left(N_{s}+1\right)$, where $\left\{\lambda_{i}, 1 \leq i \leq N_{\eta}\right\}$ are the $N_{\eta}$ most influencing eigenvalues, and where $\left\{\mathbf{u}_{i}, 1 \leq i \leq N_{\eta}\right\}$ are the associated eigenfunctions of the covariance matrix $\left[C_{\mathbf{X X}}\right]$ of $\mathbf{X}$. The random coefficients $\left\{\eta_{i}, 1 \leq i \leq N_{\eta}\right\}$ are uncorrelated, centered and normalized random variables such that $\eta_{i}=\lambda_{i}^{-1 / 2} \mathbf{X}^{T} \mathbf{u}_{i}$ for $i=1, \ldots, N_{\eta}$. Introducing the $\left(4\left(N_{s}+1\right) \times N_{\eta}\right)$ real matrix $[U]$ defined by

$$
[U]=\left[\mathbf{u}_{1} \ldots \mathbf{u}_{N_{\eta}}\right] \quad, \quad[U]^{T}[U]=\left[I_{N_{\eta}}\right],
$$

in which $\left[I_{N_{\eta}}\right]$ is the unity matrix, the diagonal matrix $[\lambda]$ whose diagonal entries are $\lambda_{1}, \ldots, \lambda_{N_{\eta}}$, and the random vector $\boldsymbol{\eta}$ such that, $\boldsymbol{\eta}=\left(\eta_{1}, \ldots, \eta_{N_{\eta}}\right)$, then random vector $\mathbf{X}$ can be rewritten as $\mathbf{X} \simeq[U][\lambda]^{1 / 2} \boldsymbol{\eta}$. Introducing $[Q]=[U][\lambda]^{1 / 2}$, it yields

$$
\mathbf{X} \simeq[Q] \boldsymbol{\eta}
$$

Nevertheless, as explained in Section 2, we are interested in constructing a stochastic model adapted to a given stretch of track. The objective of this adapted stochastic model that has to be constructed is to take into account uncertainties induced by (i) measurement noise associated with local measurements $\mathbf{x}_{\tau_{0}}^{\text {meas }}, \mathbf{x}_{\tau_{1}}^{\text {meas }}, \mathbf{x}_{\tau_{2}}^{\text {meas }}, \ldots$, 
and (ii) the local variability of the given track stretch in order to decrease the "statistical distance" between the global stochastic model and the local measurements.

\subsection{Local stochastic modeling}

The local stochastic modeling aims at adapting the global stochastic modeling of the track irregularities to a given stretch of track. For constructing such an adapted stochastic model, the proposed method consists in introducing a random noise $\mathbf{B}$ in the modeling of the track irregularities. The spatial properties of this random noise will be induced by the global stochastic model. The intensity of the statistical fluctuations of the introduced noise, which are controlled by a hyperparameter $\boldsymbol{\delta}_{\tau_{0}}$, will be identified at long time $\tau_{0}$ using measurement $\mathbf{x}_{\tau_{0}}^{\text {meas }}$. It is then assumed that the optimal value for the intensity of the statistical fluctuations of the input noise, is representative of the level of uncertainties (measurement noise and variability) for all the values of the long time of the given track stretch. This assumption seems to be reliable, because it is believable that the measurement noise and the variability of the track irregularities remain the same between two successive maintenance operations. Moreover, identifying the local stochastic model at each long time could prevent from visualizing the long-term evolution of the track irregularities between two successive track measurements, because it could regard the long-term evolution of the track irregularities in the random noise. For $k=1, \ldots, 4, \mathbf{X}^{k}$ is the random vector of dimension $N_{s}+1$ defined (using Equation (7)) as

$$
\mathbf{X}^{k}=\left[Q^{k}\right] \boldsymbol{\eta},
$$

in which the $\left(\left(N_{s}+1\right) \times N_{\eta}\right)$ real matrix $\left[Q^{k}\right]$ is extracted from matrix $[Q]$. Introducing the input noise $\mathbf{B}\left(\boldsymbol{\delta}_{\tau_{0}}\right)=\left(B^{1}\left(\delta_{\tau_{0}}^{1}\right), B^{2}\left(\delta_{\tau_{0}}^{2}\right), B^{3}\left(\delta_{\tau_{0}}^{3}\right), B^{4}\left(\delta_{\tau_{0}}^{4}\right)\right)$ that depends on hyperparameter $\boldsymbol{\delta}_{\tau_{0}}$, the proposed adapted stochastic model is written as

$$
\widetilde{\mathbf{X}}^{k}\left(\delta_{\tau_{0}}^{k}\right)=\mathbf{X}^{k}+\mathbf{B}^{k}\left(\delta_{\tau_{0}}^{k}\right) .
$$

For preserving the spatial properties of the track irregularities present in matrix $\left[Q^{k}\right]$, for $k=1,2,3,4$, random vector $\mathbf{B}^{k}\left(\delta_{\tau_{0}}^{k}\right)$ is such that

$$
\mathbf{B}^{k}\left(\delta_{\tau_{0}}^{k}\right)=\delta_{\tau_{0}}^{k}\left[Q^{k}\right] \mathbf{G}^{k}
$$

in which $\boldsymbol{\delta}_{\tau_{0}}=\left(\delta_{\tau_{0}}^{1}, \delta_{\tau_{0}}^{2}, \delta_{\tau_{0}}^{3}, \delta_{\tau_{0}}^{4}\right)$ is the vector-valued hyperparameter allowing the intensity of the statistical fluctuations to be controlled, and which has to be identified for each track stretch using experimental data at $\tau=\tau_{0}$. For fixed $k, \mathbf{G}^{k}$ is a $\mathbb{R}^{N_{\eta}}$-valued random vector noise. In the model proposed, $\mathbf{G}=\left(\mathbf{G}^{1}, \mathbf{G}^{2}, \mathbf{G}^{3}, \mathbf{G}^{4}\right)$ is chosen as a $\mathbb{R}^{4 N_{\eta}}$-valued Gaussian second-order centered random variable, defined on the probability space $\left(\Theta^{\prime}, \mathcal{F}^{\prime}, \mathcal{P}^{\prime}\right)$, for which its covariance matrix is the unity matrix.

From Equations (8), (9), and (10), the adapted stochastic model can be rewritten as

$$
\widetilde{\mathbf{X}}^{k}\left(\delta_{k}\right)=\left[Q^{k}\right]\left(\boldsymbol{\eta}+\delta_{\tau_{0}}^{k} \mathbf{G}^{k}\right) \quad, \quad k=1,2,3,4 .
$$

The optimal value $\boldsymbol{\delta}_{\tau_{0}}^{\text {opt }}$ of hyperparameter $\boldsymbol{\delta}_{\tau_{0}}$ is estimated by using the maximum log-likelihood method with experimental data applied to the observation random 
vector $\mathbf{W}(\boldsymbol{\delta})=\left(W^{1}\left(\delta^{1}\right), W^{2}\left(\delta^{2}\right), W^{3}\left(\delta^{3}\right), W^{4}\left(\delta^{4}\right)\right)$, in which

$$
W^{k}\left(\delta^{k}\right)=\frac{\left\|\widetilde{\mathbf{X}}^{k}\left(\delta^{k}\right)\right\|}{E\left\{\left\|\mathbf{X}^{k}\right\|\right\}}
$$

where $\mathbf{X}^{k}=\widetilde{\mathbf{X}}^{k}(0)$, and where $\|\cdot\|$ is the Euclidean norm of the global stochastic model $\mathbf{X}^{k}$ (see [3]). The experimental observation vector $\mathbf{w}_{\tau_{0}}^{\text {meas }}=$ $\left(w_{\tau_{0}}^{\text {meas }, 1}, w_{\tau_{0}}^{\text {meas, }, 2}, w_{\tau_{0}}^{\text {meas }, 3}, w_{\tau_{0}}^{\text {meas, } 4}\right)$ that corresponds to the experimental measurement of $\mathbf{W}(\boldsymbol{\delta})$ at long time $\tau_{0}$ is such that

$$
w_{\tau_{0}}^{\text {meas }, k}=\frac{\left\|\mathbf{x}_{\tau_{0}}^{\text {meas }, k}\right\|}{E\left\{\left\|\mathbf{X}^{k}\right\|\right\}}, 1 \leq k \leq 4
$$

Let $\mathcal{L}_{\mathbf{W}}\left(\mathbf{w}_{\tau_{0}}^{\text {meas }} ; \boldsymbol{\delta}\right)=\log p_{\mathbf{W}}\left(\mathbf{w}_{\tau_{0}}^{\text {meas }} ; \boldsymbol{\delta}\right)$ be the $\log$-likelihood function in which $p_{\mathbf{W}}\left(\mathbf{w}_{\tau_{0}}^{\text {meas }} ; \boldsymbol{\delta}\right)$ is the value of the probability density function $\mathbf{w} \mapsto p_{\mathbf{W}}(\mathbf{w} ; \boldsymbol{\delta})$ of random vector $\mathbf{W}$ for $\mathbf{w}=\mathbf{w}_{\tau_{0}}^{\text {meas }}$. The optimal value $\boldsymbol{\delta}_{\tau_{0}}^{\text {opt }}$ is then identified solving the following optimization problem:

$$
\boldsymbol{\delta}_{\tau_{0}}^{\text {opt }}=\arg \max _{\boldsymbol{\delta}}\left\{\mathcal{L}_{\mathbf{W}}\left(\mathbf{w}_{\tau_{0}}^{\text {meas }} ; \boldsymbol{\delta}\right)\right\}
$$

The quantity $p_{\mathbf{W}}\left(\mathbf{w}_{\tau_{0}}^{\text {meas }} ; \boldsymbol{\delta}\right)$ is computed using independent realizations of $\mathbf{W}$ generated with the adapted stochastic model, and fitted by using the multivariate Gaussian kernel method (see for instance [17, 18]). As an illustration, and for $k=2$, Figure 1 displays the variation of the marginal probability density function $(\mathrm{PDF}) w^{k} \mapsto p_{W^{k}\left(\delta^{k}\right)}\left(w_{\tau_{0}}^{k} ; \delta^{k}\right)$ of random variable $W^{k}\left(\delta^{k}\right)$ as a function of $\delta^{k}$.

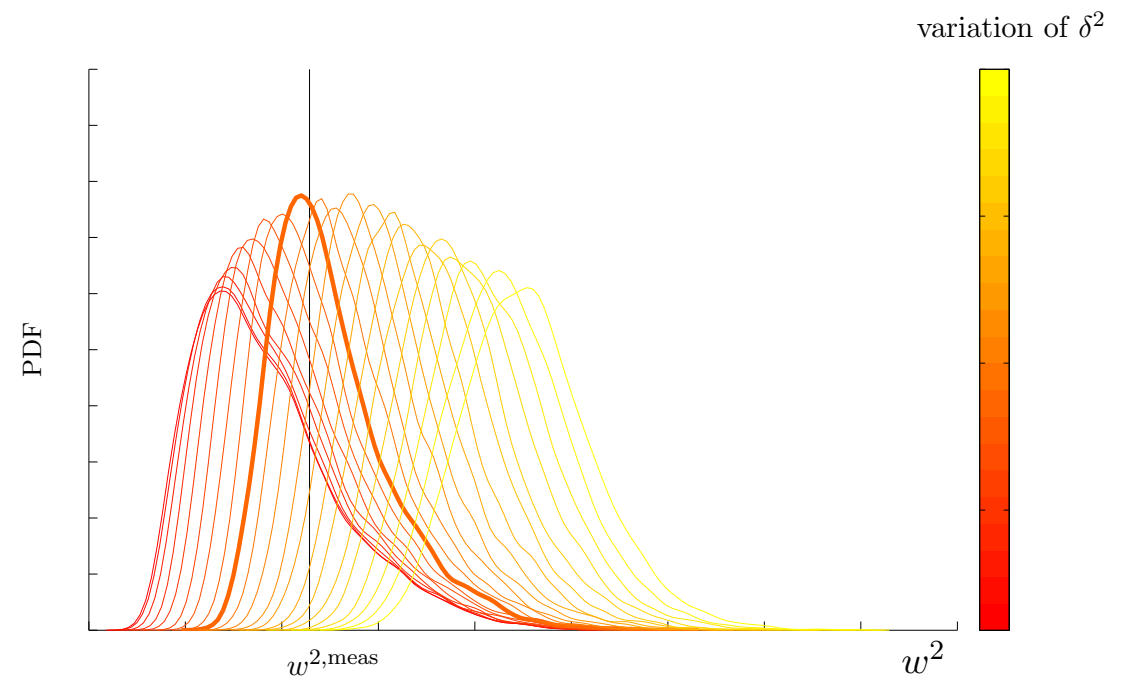

Figure 1. Graphs of the PDF, $w^{2} \mapsto p_{W^{2}\left(\delta^{2}\right)}\left(w^{2} ; \delta^{2}\right)$, as a function of $\delta^{2}$ (the bold line is obtained for $\left.\delta^{2}=\delta_{\tau_{0}}^{\mathrm{opt}, 2}\right)$

The adapted stochastic model of the track irregularities for a given stretch of track at long time $\tau$ is constructed as follows. At long time $\tau$, the measurement of the track stretch is $\mathbf{x}_{\tau}^{\text {meas }}=\left(\mathbf{x}_{\tau}^{\text {meas }, 1}, \mathbf{x}_{\tau}^{\text {meas }, 2}, \mathbf{x}_{\tau}^{\text {meas }, 3}, \mathbf{x}_{\tau}^{\text {meas }, 4}\right)$. First, we have to calculate the realization $\boldsymbol{\eta}_{\tau}^{\text {meas }}$ of random vector $\boldsymbol{\eta}$ of the generalized coordinates of the global stochastic model introduced in Equation (5) and rewritten as Equa$\operatorname{tion}(7)$. This realization is calculated as the projection of the measurement $\mathbf{x}_{\tau}^{\text {meas }}$ 
on the global stochastic model, which yields

$$
\boldsymbol{\eta}_{\tau}^{\text {meas }}=[\lambda]^{-1}[Q]^{T} \mathbf{x}_{\tau}^{\text {meas }}
$$

At long time $\tau$, the adapted stochastic model is then defined as

$$
\widetilde{\mathbf{X}}_{\tau}^{k}\left(\delta_{\tau_{0}}^{\mathrm{opt}, k}\right)=\left[Q^{k}\right]\left(\boldsymbol{\eta}_{\tau}^{\mathrm{meas}}+\delta_{\tau_{0}}^{\mathrm{opt}, k} \mathbf{G}^{k}\right) \quad, \quad k=1,2,3,4
$$

As an illustration, a realization $\mathbf{X}_{\tau_{0}}^{2}\left(\theta^{\prime}\right), \theta^{\prime} \in \Theta^{\prime}$, of $\mathbf{X}_{\tau_{0}}^{2}$ and the confidence region at $90 \%$ of $\widetilde{\mathbf{X}}_{\tau_{0}}^{2}\left(\delta_{\tau_{0}}^{\text {opt }, 2}\right)$ are displayed in Figure 2 . For confidentiality reasons, numerical values are not displayed. It can be noticed that the geometrical and physical properties of the irregularities are preserved with the identified adapted stochastic modeling. Finally, the local stochastic model that has been constructed allows for adapting the global stochastic model of the track irregularities to a given track stretch. The statistical properties of the track irregularities of the given track stretch are preserved by the local stochastic model. The local stochastic model also enables to generate realizations of the track irregularities, by generating realizations of random vector $\mathbf{G}$ from the probability space $\left(\Theta^{\prime}, \mathcal{F}^{\prime}, \mathcal{P}^{\prime}\right)$.

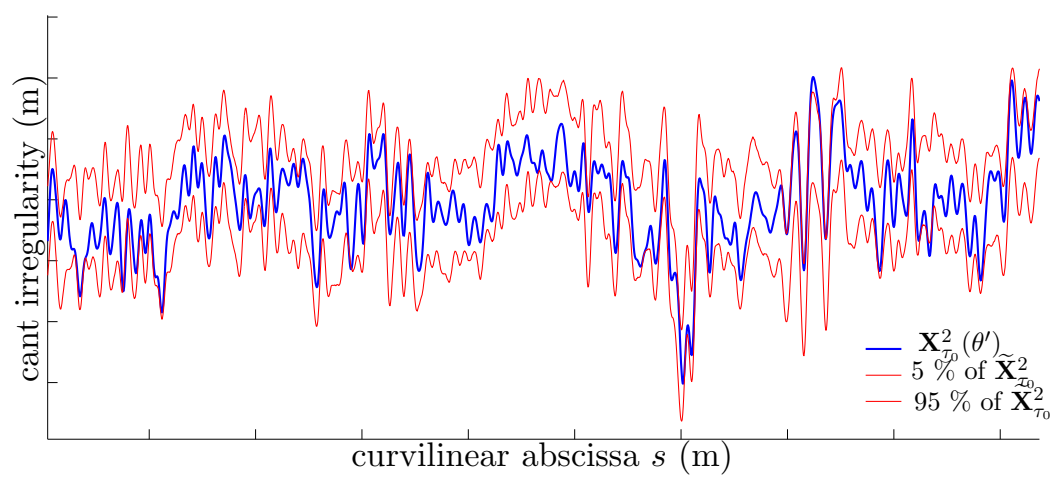

Figure 2. Irregularity $\mathbf{X}_{\tau_{0}}^{2}\left(\theta^{\prime}\right)$ and confidence region at $90 \%$ of $\widetilde{\mathbf{X}}_{\tau_{0}}^{2}\left(\delta^{\text {opt }, 2}\right)$ for the given track stretch. Upper line $\left(95 \%\right.$ of $\left.\widetilde{\mathbf{X}}_{\tau_{0}}^{2}\right)$, bold line $\left(\mathbf{X}_{\tau_{0}}^{2}\left(\theta^{\prime}\right), \theta^{\prime} \in \Theta^{\prime}\right)$, and lower line $\left(5 \%\right.$ of $\left.\widetilde{\mathbf{X}}_{\tau_{0}}^{2}\right)$.

\section{Stochastic modeling of model uncertainties in the train computational model}

\subsection{Definition of dynamic indicators}

The dynamic response of the train on a given track stretch is simulated using the track measurements. In this section, nine indicators are defined, which are denoted by $c_{j}$ for $j=1, \ldots, 9$, in order to assess the train dynamics on this track stretch. Those indicators are computed from forces and accelerations measured or simulated at different locations of the train, for a given track stretch of length $S$. They are based on criteria described in norm UIC 518 [19] for the certification of railway vehicles. From a complete and detailed analysis performed with experimental measurements, the following observations have been retained for defining the indicators related to the French high-speed trains. For $s$ belonging to $[0, S]$, the chosen accelerations are

- the lateral acceleration of the first bogie of the train, denoted by $A_{1}(s)$, 
- the vertical acceleration of the first bogie of the train, denoted by $A_{2}(s)$,

- the lateral acceleration of the third bogie of the train, denoted by $A_{3}(s)$,

- the lateral acceleration of the second coach of the train, denoted by $A_{4}(s)$.

The longitudinal direction is the $(O x)$-axis and corresponds to the track axis. The lateral direction is the $(O y)$-axis and corresponds to the direction that is horizontal and perpendicular to $(O x)$. The vertical direction is the $(O z)$-axis and is oriented upwards. For the experimental measurements, the accelerometers are not located at the center of gravity of the bodies (bogies and coaches). In order to compute in the simulation what has been experimentally measured, the simulated accelerations have to take into account the momentum due to the off-centering of the accelerometers. For lateral accelerations, we have:

$$
A_{j}(s)=\ddot{y}_{j}(s)+\ddot{w}_{j}^{x}(s) l_{j}^{x}-\ddot{w}_{j}^{z}(s) l_{j}^{z} \quad, \quad s \in[0, S] \quad, \quad j=1,3,4,
$$

where $\ddot{y}_{j}(s)$ is the lateral acceleration of the considered bodies, $l_{j}^{x}$ and $l_{j}^{z}$ are the distances between the center of gravity of the bodies and the sensor in the longitudinal and vertical directions respectively, and where $\ddot{w}_{j}^{x}$ and $\ddot{w}_{j}^{z}, j=1,3,4$, are the angular accelerations about the longitudinal and vertical axes respectively. For the vertical acceleration $A_{2}$,

$$
A_{2}(s)=\ddot{z}_{2}(s)-\ddot{w}_{2}^{x}(s) l_{2}^{x}+\ddot{w}_{2}^{y}(s) l_{2}^{y} \quad, \quad s \in[0, S]
$$

in which $\ddot{z}_{2}(s)$ is the vertical acceleration of the first bogie, $l_{2}^{y}$ is the distance between the center of gravity of the bogie and the accelerometer in the lateral direction, and where $\ddot{w}_{2}^{y}$ is the angular acceleration about the lateral axis. A fourth-order bandpass linear filter is used in order to restrict the signal into the frequency band for which the train model is relevant for the accelerations.

Five more dynamic outputs are computed from wheel-rail contact forces. They depend on lateral contact forces $Y$ and on vertical contact forces $Q$, which are computed for the wheel-rail contact on the right side (represented by index $R$ ) and on the left side (represented by index $L$ ) of a wheelset. For a curvilinear abscissa $s$, these indicators are defined as

- the sum of lateral forces on the ninth wheelset, defined by $A_{5}(s)=Y_{9}^{R}(s)+Y_{9}^{L}(s)$,

- the sum of vertical forces on the first wheelset, defined by $A_{6}(s)=Q_{1}^{R}(s)+Q_{1}^{L}(s)$,

- the sum of vertical forces on the second wheelset, defined by $A_{7}(s)=Q_{2}^{R}(s)+Q_{2}^{L}(s)$

- the sum of vertical forces on the tenth wheelset, defined by $A_{8}(s)=Q_{10}^{R}(s)+Q_{10}^{L}(s)$,

- the difference of vertical forces on the tenth wheelset, defined by $A_{9}(s)=Q_{10}^{R}(s)-Q_{10}^{L}(s)$.

The signals are filtered in order to restrict the analysis to the frequency band for which the train computational model is relevant for the forces. For the measured forces, the filter is a fourth-order low-pass linear filter, according to [19]. Moreover, for $A_{5}$, the signal is filtered by computing its sliding average over two meters of the track.

Let $N_{c}=9$ be the number of indicators. The components of the indicators vector $\mathbf{c}=\left(c_{1}, \ldots, c_{N_{c}}\right)$ of the dynamic indicators are defined by

$$
c_{j}=\max _{s \in[0, S]}\left|A_{j}(s)\right| \quad, \quad j=1, \ldots, N_{c} .
$$




\subsection{Experimental measurements and numerical simulation of the dynamic response of the train}

In order to characterize the model uncertainties in the computational model of the train dynamics, we need to compare the indicators vector $\mathbf{c}^{\mathrm{sim}}=\left(c_{1}^{\mathrm{sim}}, \ldots, c_{9}^{\mathrm{sim}}\right)$ obtained by the simulation, with experimentally measured indicators vector $\mathbf{c}^{\exp }=$ $\left(c_{1}^{\exp }, \ldots, c_{9}^{\exp }\right)$ for the deterministic track geometry of each stretch of track. To achieve this goal, the accelerations and the forces that are needed to compute the indicators are experimentally measured and numerically simulated on the same track. A measuring train is equipped with sensors and travels across a high-speed line at several speeds and with different loads for its second coach. Five measurement campaigns have been performed on the track, measuring the dynamic response of the train. The accelerations have been measured on the bogies and the coaches of the train using accelerometers. The forces applied on the train wheels are computed using the wheels strain, which are measured using strain gauges fixed in the wheels.

The measurements are performed for $\nu$ stretches of the track. For each track stretch denoted by $\ell \in\{1, \ldots, \nu\}$, the speed of the train, the track geometry $\mathbf{x}^{\exp , \ell}$, and the indicators vector $\mathbf{c}^{\mathrm{exp}^{\ell} \ell}$ are simultaneously measured and recorded (the experimental indicators vector is deduced from the measured accelerations and forces). It is assumed that $\mathbf{c}^{\exp , 1}, \ldots, \mathbf{c}^{\exp , \nu}$ are $\nu$ independent realizations of a random indicators vector denoted as $\mathbf{C}^{\exp }$.

Then, the dynamic response of the train, on the track that has been measured, is numerically simulated using the experimental conditions as an input of the simulation. The track geometry used in input is the deterministic track geometry measured during the measurement campaigns (without using the adapted stochastic model of Section 3). The international norm [19] imposes an output signal sample frequency $F_{s}$ of at least $200 \mathrm{~Hz}$. To have the same sample frequency as the measurement, the simulation sample frequency is chosen at $400 \mathrm{~Hz}$. The input speed is the recorded speed of the measuring train and the input vehicle is the computational model of the measuring train, which is a TGV Duplex. This computational model is a multibody model with two levels of nonlinear suspensions and with a nonlinear wheel-rail contact model, for which the physical parameters have been identified by using an inverse method and experimental measurements [20]. In the computational model, despite a precise identification, some uncertainties remain, because of the high number of physical parameters (more than 800). Uncertainties in the computational model are also due to the multibody modeling, to the modeling of nonlinear suspensions or of the nonlinear wheel-rail contact, and to numerical errors. Because the software Vampire is considered as a blackbox, we chose to take into account the model uncertainties by introducing an output noise in the construction of the indicators. For each measured track stretch $\ell=1, \ldots, \nu$ with $\nu=937$, the train dynamics on the stretch is simulated with the computational model and indicators vector $\mathbf{c}^{\operatorname{sim}, \ell}$ are computed (as defined in Section 4.1). It is assumed that $\mathbf{c}^{\operatorname{sim}, 1}, \ldots, \mathbf{c}^{\operatorname{sim}, \nu}$ are $\nu$ independent realizations of a random indicators vector denoted by $\mathbf{C}^{\text {sim }}$. Despite the use of an advanced computational model for the train dynamics, the models of the suspensions of the train and of the wheel-rail contact differently behave in tangent track or in curved track. Thus it can be assumed that the model uncertainties (which take into account uncertainties in the modeling of the suspensions and of the wheel-rail contact) in the computational model of the train dynamics depend on the track curvature. Consequently, the stretches of track are decomposed in four curvature classes:

- tangent track, for which the horizontal curvature is zero, 


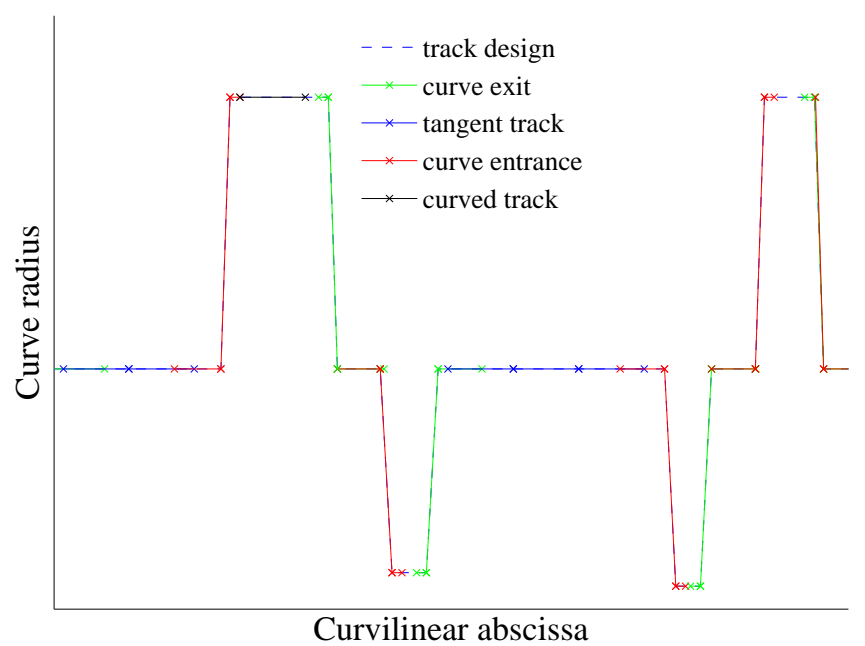

Figure 3. Division of a track section into track stretches of same length $S$ with four curvature types.

- curved track, for which the horizontal curvature is constant and non zero,

- curve entrance (transition curve approaching to a circular curve), for which the absolute value of the curvature is linearly increasing,

- curve exit (transition curve receding from a circular curve), for which the absolute value of the curvature is linearly decreasing.

We have $\nu_{A}=341$ stretches in tangent track, $\nu_{C}=311$ in curved track, $\nu_{E C}=114$ in curve entrance, and $\nu_{S C}=132$ in curve exit. As an illustration, Figure 3 diplays the division of a track section into stretches of track of same length $S$ with four curvature types.

\subsection{Model uncertainties induced by modeling errors in the train computational model}

\subsubsection{Stochastic modeling}

The stochastic model of model uncertainties induced by modeling errors [21] in the train computational model is constructed in introducing a multiplicative output noise in the simulated indicators vector $\mathbf{C}^{\operatorname{sim}}$. To characterize this output noise, a random vector $\mathbf{B}^{\text {out }}$ is introduced, which is statistically independent of the $\mathbf{C}^{\text {sim }}$, and consequently has been defined on another probability space denoted by $\left(\Theta^{\prime \prime}, \mathcal{F}^{\prime \prime}, \mathcal{P}^{\prime \prime}\right)$. The random indicators vector that includes model uncertainties is denoted by $\mathbf{C}^{\text {mod }}=\left(C_{1}^{\text {mod }}, \ldots, C_{N_{c}}^{\text {mod }}\right)$ and is then defined by

$$
C_{j}^{\text {mod }}\left(\theta^{\prime}, \theta^{\prime \prime}\right)=C_{j}^{\text {sim }}\left(\theta^{\prime}\right) \exp \left(B_{j}^{\text {out }}\left(\theta^{\prime \prime}\right)\right), j=1, \ldots, N_{c}, \theta^{\prime} \in \Theta^{\prime}, \theta^{\prime \prime} \in \Theta^{\prime \prime},
$$

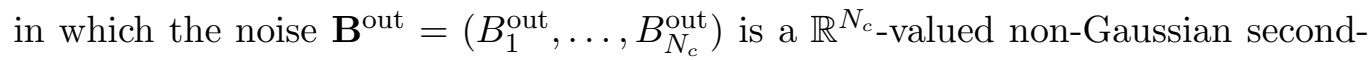
order random vector, which must be identified in comparing the random indicators vector $\mathbf{C}^{\text {mod }}$ with the corresponding random indicators vector $\mathbf{C}^{\exp }$ for which measurements have been carried out for the $\nu$ track stretches. Random vector $\mathbf{B}^{\text {out }}$ is 
classically written as

$$
\mathbf{B}^{\text {out }}=\underline{\mathbf{b}}+\sum_{j=1}^{N_{c}} \sqrt{\lambda_{j}} \eta_{j} \varphi^{j}
$$

in which $\underline{\mathbf{b}}=E\left\{\mathbf{B}^{\text {out }}\right\}$ is the mean value, and where $\lambda_{1}, \ldots, \lambda_{N_{c}}$ and $\varphi^{1}, \ldots, \varphi^{N_{c}}$ are the positive eigenvalues and the orthonormal eigenvectors of the covariance matrix $\left[C_{\mathbf{B}^{\text {out }}}\right]=E\left\{\left(\mathbf{B}^{\text {out }}-\underline{\mathbf{b}}\right)\left(\mathbf{B}^{\text {out }}-\underline{\mathbf{b}}\right)^{T}\right\}$ that will be estimated with the measurements. The non-Gaussian random vector $\boldsymbol{\eta}=\left(\eta_{1}, \ldots, \eta_{N_{c}}\right)$, for which that components are such that $\eta_{j}=\lambda_{j}^{-1 / 2}\left(\mathbf{B}^{\text {out }}-\underline{\mathbf{b}}\right)\left(\boldsymbol{\varphi}^{j}\right)^{T}$, is such that $E\{\boldsymbol{\eta}\}=0$ and $E\left\{\boldsymbol{\eta} \boldsymbol{\eta}^{T}\right\}=\left[I_{N_{c}}\right]$ (the unit matrix). In order to represent a family of parameterized probability distributions of the non-Gaussian random vector $\boldsymbol{\eta}$, the following finite polynomial chaos expansion (PCE) (see for instance [21-23]) is introduced,

$$
\boldsymbol{\eta}=\sum_{n=0}^{N} \mathbf{y}^{(n)} \psi_{\boldsymbol{\alpha}^{(n)}}(\boldsymbol{\xi})
$$

in which $\boldsymbol{\xi}=\left(\xi_{1}, \ldots, \xi_{N_{g}}\right)$ is a normalized Gaussian random vector of a dimension $N_{g} \leq N_{c}$. Consequently, the components $\xi_{1}, \ldots, \xi_{N_{g}}$ are independent normalized Gaussian real-valued random variables, and $\psi_{\boldsymbol{\alpha}^{(0)}}(\boldsymbol{\xi}), \ldots, \psi_{\boldsymbol{\alpha}^{(N)}}(\boldsymbol{\xi})$ are the multivariate orthonormal normalized Hermite polynomials (the polynomial chaos), and where $N, N_{g}$ and the vectors $\mathbf{y}^{(0)}, \ldots, \mathbf{y}^{(N)}$ in $\mathbb{R}^{N_{c}}$ have to be identified. For $n=0, \ldots, N$, the multi-index $\boldsymbol{\alpha}^{(n)}$ is the vector of integers defined by $\boldsymbol{\alpha}^{(n)}=\left(\alpha_{1}^{n}, \ldots, \alpha_{N_{g}}^{n}\right) \in \mathbb{N}^{N_{g}}$. If $p$ denotes the maximal degree of the polynomials, we have $0 \leq \alpha_{1}^{n}+\ldots+\alpha_{N_{g}}^{n} \leq p$, and integer $N$ is such that

$$
N=\frac{\left(N_{g}+p\right) !}{\left(N_{g} ! p !\right)}
$$

The polynomial chaos $\psi_{\boldsymbol{\alpha}^{(n)}}(\boldsymbol{\xi})$ are such that

$$
\psi_{\boldsymbol{\alpha}^{(n)}}(\boldsymbol{\xi})=\psi_{\alpha_{1}(n)}\left(\xi_{1}\right) \times \ldots \times \psi_{\alpha_{N_{g}}(n)}\left(\xi_{N_{g}}\right) \quad, \quad n=1, \ldots, N
$$

with $\psi_{\boldsymbol{\alpha}^{(0)}}(\boldsymbol{\xi})=1$ and satisfy the orthonormal property,

$$
E\left\{\psi_{\boldsymbol{\alpha}^{(n)}}(\boldsymbol{\xi}) \psi_{\boldsymbol{\alpha}^{(k)}}(\boldsymbol{\xi})\right\}=\delta_{n k},
$$

where $\delta_{n k}$ is the Kronecker symbol, such that $\delta_{n k}=1$ if $n=k$ and $\delta_{n k}=0$ otherwise.

\subsubsection{Identification}

Taking into account the hypotheses and the notations introduced in Section 4.2, and since, for all fixed $\ell$ and $j$, we have $c_{j}^{\text {sim, } \ell}>0$, the realization $B_{j}^{\text {out }}\left(\theta_{\ell}^{\prime \prime}\right)$ of the random variable $B_{j}^{\text {out }}$, which is rewritten as $b_{j}^{\exp , \ell}$, can be calculated by using Equation (20),

$$
b_{j}^{\exp , \ell}=\ln \left(\frac{c_{j}^{\exp , \ell}}{c_{j}^{\operatorname{sim}, \ell}}\right) \quad, \quad j=1, \ldots, N_{c} \quad, \quad \ell=1, \ldots, \nu .
$$


For numerical considerations, each component $b_{j}^{\exp , \ell}$ of the vector $\mathbf{b}^{\exp , \ell}=$ $\left(b_{1}^{\exp , \ell}, \ldots, b_{N_{c}}^{\exp , \ell}\right)$ could be normalized by $\max _{\ell}\left|b_{j}^{\exp , \ell}\right|$. The following estimations of mean value $\underline{\mathbf{b}}$ and covariance matrix $\left[C_{\mathbf{B}^{\text {out }}}\right]$ (defined in Section 4.3 .1 ) are introduced,

$$
\underline{\mathbf{b}} \simeq \underline{\mathbf{b}}^{\exp }=\frac{1}{\nu} \sum_{\ell=1}^{\nu} \mathbf{b}^{\exp , \ell} \quad, \quad\left[C_{\mathbf{B}^{\text {out }}}\right] \simeq \frac{1}{\nu-1} \sum_{\ell=1}^{\nu}\left(\mathbf{b}^{\exp , \ell}-\underline{\mathbf{b}}^{\exp }\right)\left(\mathbf{b}^{\exp , \ell}-\underline{\mathbf{b}}^{\exp }\right)^{T} .
$$

The independent realizations $\boldsymbol{\eta}^{\exp , \ell}=\left(\eta_{1}^{\exp , \ell}, \ldots, \eta_{N_{c}}^{\exp , \ell}\right)$ of random vector $\boldsymbol{\eta}$ are then calculated by $\eta_{j}^{\exp , \ell}=\lambda_{j}^{-1 / 2}\left(\mathbf{b}^{\exp , \ell}-\underline{\mathbf{b}}^{\exp }\right)\left(\boldsymbol{\varphi}^{j}\right)^{T}$.

Let $\mathbf{h} \mapsto p_{\boldsymbol{\eta}}(\mathbf{h} ;[y])$ be the probability density function of random vector $\boldsymbol{\eta}$ defined by Equation (22) and depending on the matrix $[y]=\left[\mathbf{y}^{0}, \ldots, \mathbf{y}^{N}\right]$ that belongs to the set $\mathbb{M}_{N_{c}, N}$ of all the real $\left(N_{c} \times N\right)$ matrices and that verifies the constraints $[y][y]^{T}=\left[I_{N_{c}}\right]$. Consequently, $[y]$ must be identified in the admissible set defined by

$$
\mathcal{C}_{[y]}=\left\{[y] \in \mathbb{M}_{N_{c}, N} \quad \text { such that } \quad[y][y]^{T}=\left[I_{N_{c}}\right]\right\} .
$$

For each fixed $[y]$ and fixed $\mathbf{h}, p_{\boldsymbol{\eta}}(\mathbf{h} ;[y])$ is estimated by using samples calculated with Equation (22) and the Gaussian kernel estimation method. Matrix [y] is identified by the method described in [4, 14], which is briefly summarized hereinafter.

For integers $N_{g}$ and $p$ fixed, the optimal value $\left[y^{\text {opt }}\left(N_{g}, p\right)\right]$ of $[y]$ is calculated in solving the optimization problem (maximum log-likelihood method),

$$
\left[y^{\text {opt }}\left(N_{g}, p\right)\right]=\arg \max _{[y] \in \mathcal{C}_{[y]}} \sum_{\ell=1}^{\nu} \ln \left(p_{\boldsymbol{\eta}}\left(\boldsymbol{\eta}^{\exp , \ell} ;[y]\right)\right) .
$$

The optimal values of integers $N_{g}$ and $p$ are calculated in order to minimize the value of $N$ defined by Equation (23), while respecting the condition given by the dimension of $[y]$, which is written as

$$
\frac{\left(N_{g}+p\right) !}{\left(N_{g} ! p !\right)} \geq \frac{N_{c}+1}{2}
$$

Let $\mathcal{C}_{N}$ be the admissible set of the couples $\left(N_{g}, p\right)$ of integers, which satisfy Equation (30). The optimal value $\left(N_{g}^{\mathrm{opt}}, p^{\mathrm{opt}}\right)$ of $\left(N_{g}, p\right)$ are thus calculated as

$$
\left(N_{g}^{\mathrm{opt}}, p^{\mathrm{opt}}\right)=\arg \min _{\left(N_{g}, p\right) \in \mathcal{C}_{N}} \operatorname{err}\left(N_{g}, p\right)
$$

in which the error function is written as

$$
\operatorname{err}\left(N_{g}, p\right)=\sum_{j=1}^{N_{c}} \int_{\mathrm{BI}_{j}}\left|\log _{10}\left(p_{\eta_{j}^{\exp }}\left(h_{j}\right)\right)-\log _{10}\left(p_{\eta_{j}}\left(h_{j} ;\left[y^{\mathrm{opt}}\left(N_{g}, p\right)\right]\right)\right)\right| d h_{j},
$$

in which $p_{\eta_{j}^{\text {exp }}}$ is the probability density function estimated by using the Gaussian kernel estimation method and the realizations $\eta_{j}^{\text {exp }, \ell}, \ell=1, \ldots, \nu$, and where $\mathrm{BI}_{j}$ 
is the interval on which the probability density function of $\eta_{j}^{\exp }$ is higher than $\frac{1}{\nu}$,

$$
\mathrm{BI}_{j}=\left\{h_{j}, \mid, p_{\eta_{j}^{\exp }}\left(h_{j}\right) \geq \frac{1}{\nu}\right\} \quad, \quad j=1, \ldots, N_{c} .
$$

\subsubsection{Identification results}

Because the dynamic response of the train depends on the track curvature, it is assumed that the noise $\mathbf{B}^{\text {out }}$ depends on the class of the given track stretch: tangent track, curved track, curve entrance, and curve exit. The identification of the stochastic model of random vector $\mathbf{B}^{\text {out }}$ is thus carried out for each class of track stretch. For instance, in tangent track, we have $\nu=341$. For each admissible couple $\left(N_{g}, p\right) \in \mathcal{C}_{N}$, the optimal value $\left[y^{\text {opt }}\left(N_{g}, p\right)\right]$ has been computed according to Equation (29) using 100, 000 samples. Then, the error function $\left(N_{g}, p\right) \mapsto \operatorname{err}\left(N_{g}, p\right)$ is computed according to Equation (32). For example, for the tangent class of stretches, this error function, $\left(N_{g}, p\right) \mapsto \operatorname{err}\left(N_{g}, p\right)$, is plotted in Figure 4 as a function of $N_{g}$ and $p$. It can be noticed that the minimum of the function is obtained for $\left(N_{g}, p\right)=(7,2)$. For the track stretch $\ell=1$, the joint probability density

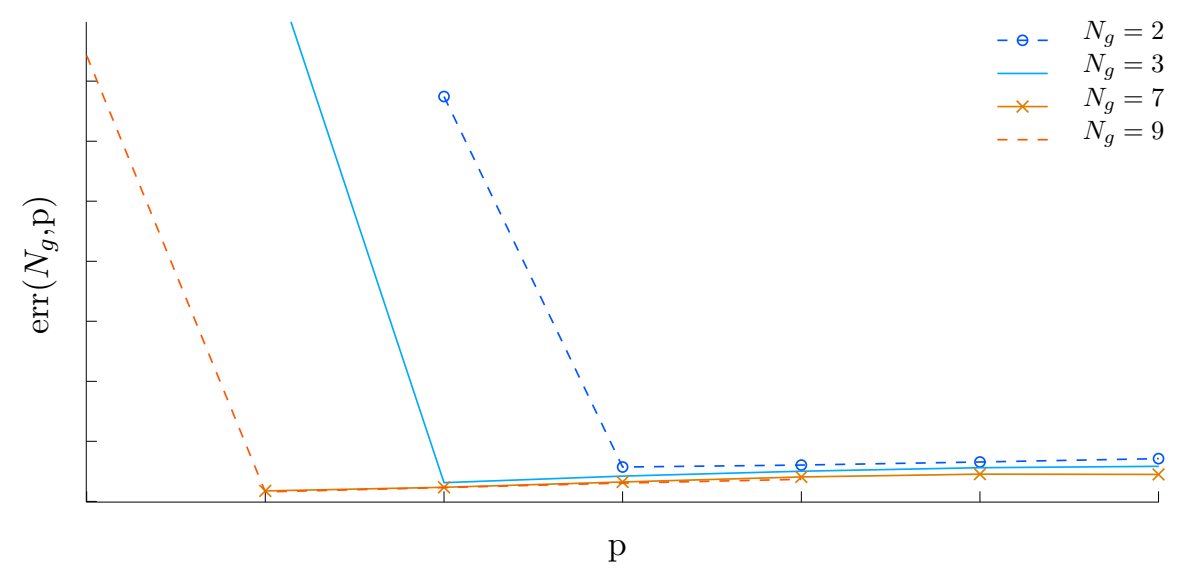

Figure 4. Error function $\operatorname{err}\left(N_{g}, p\right)$ as function of $N_{g}$ and $p$.

function of the random variables $C_{1}^{\text {mod,1 }}$ and $C_{2}^{\text {mod,1 }}$ (the first two components of $\mathbf{C}^{\text {mod,1 }}$ ) is plotted in Figure 5. In this figure, the two points corresponding to the experimental value $c_{1,2}^{\exp , 1}=\left(c_{1}^{\exp , 1}, c_{2}^{\exp , 1}\right)$ and to the simulated value $c_{1,2}^{\text {sim, } 1}=$ $\left(c_{1}^{\mathrm{sim}, 1}, c_{2}^{\mathrm{sim}, 1}\right)$ are displayed. This figure shows that the random indicators vector $\mathbf{C}^{\text {mod, } 1}$ (that takes into account the train dynamics modeling errors for a given track geometry) yields a better modeling of the experimental dynamic response of the train than $\mathbf{c}^{\text {sim,1 }}$.

\section{Results for the long-term evolution of track irregularities}

\subsection{Simulation of the dynamic response of the train}

For long time $\tau$ and for a given track stretch, the track geometry is measured by a measuring train, giving the track geometry irregularities vector $\mathbf{x}_{\tau}^{\text {meas }}=$ $\left(\mathbf{x}_{\tau}^{\text {meas, },}, \mathbf{x}_{\tau}^{\text {meas, },}, \mathbf{x}_{\tau}^{\text {meas, } 3}, \mathbf{x}_{\tau}^{\text {meas,4 }}\right)$. The optimal value $\boldsymbol{\delta}^{\text {opt }}$ of the hyperparameter $\boldsymbol{\delta}$ for this track stretch is identified using the method described in Section 3.4 (subindex $\tau_{0}$ is removed from $\boldsymbol{\delta}^{\mathrm{opt}}$ and the components of $\boldsymbol{\delta}^{\text {opt }}$ are denoted by 


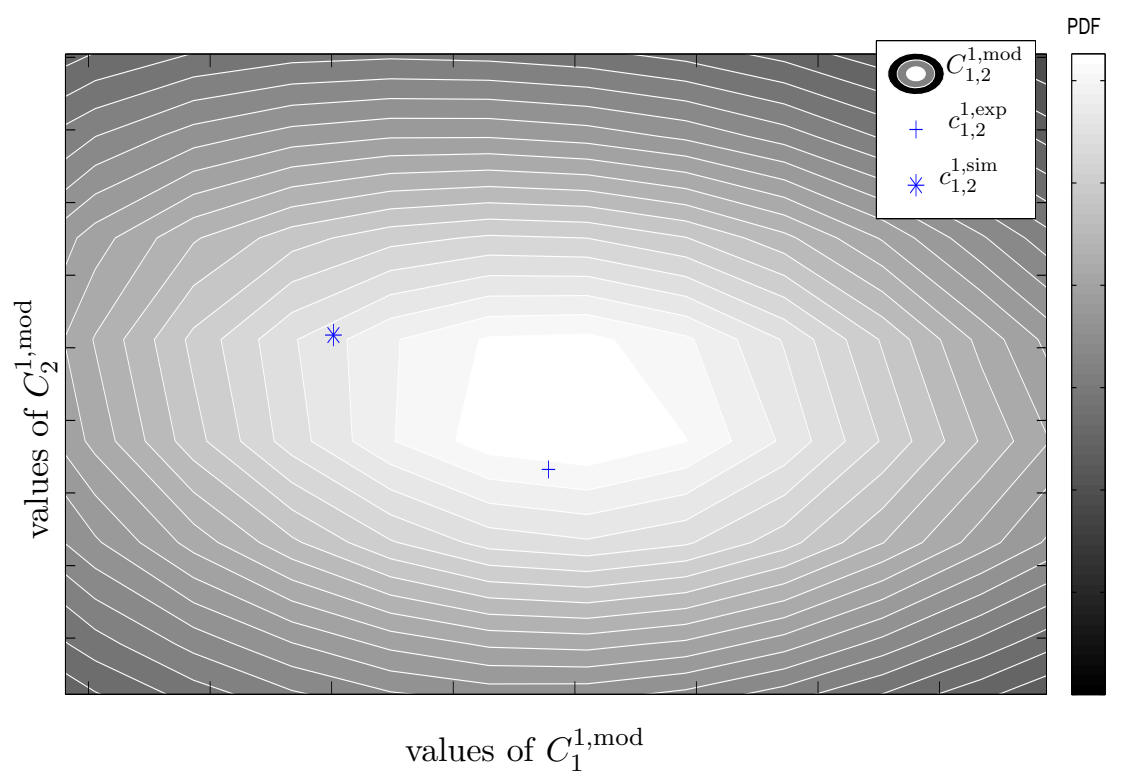

Figure 5. Position of the two points $c_{1,2}^{1, \text { exp }}$ and $c_{1,2}^{1, \text { sim }}$ into the $2 \mathrm{D}$ graph of the joint probability density function of random variables $C_{1}^{1, \bmod }$ and $C_{2}^{1, \bmod }$.

$\left.\delta^{\mathrm{opt}, k}\right)$. The admissible set for $\boldsymbol{\delta}$ is defined as the domain $[0,2]^{4}$ of $\mathbb{R}^{4}$. For estimating the optimal value, the following deterministic search algorithm is used. The identification of $\boldsymbol{\delta}^{\text {opt }}$ is made in two steps. The admissible set is partitioned in $20^{4}$ meshes corresponding to a constant step of 0.1 for each coordinate of $\boldsymbol{\delta}$. For each value of $\boldsymbol{\delta}$ corresponding to a node of the mesh, the log-likelihood is computed with 10,000 realizations of $\mathbf{W}(\boldsymbol{\delta})$. This stage allows the node of the mesh corresponding to the maximum of the log-likelihood to be identified and this node is then denoted as $\boldsymbol{\delta}_{1}=\left(\delta_{1}^{1}, \delta_{1}^{2}, \delta_{1}^{3}, \delta_{1}^{4}\right)$. Since the dimension of the admissible set of parameter $\boldsymbol{\delta}$ is small, and since each component $\delta^{k}$ belongs to a given finite interval, a deterministic algorithm can be used for solving the optimization problem defined by Equation (14). Then, the subdomain $\Pi_{k=1}^{4}\left[\delta_{1}^{k}-0.25, \delta_{1}^{k}+0.25\right]$ is explored for $\boldsymbol{\delta}$ with a precision of 0.05 for each coordinate $\left(10^{4}\right.$ meshes for the subdomain). The $\log$-likelihood is computed with 100,000 realizations for $\mathbf{W}(\boldsymbol{\delta})$. Figure 6 displays the sections (following each coordinate $\delta^{k}$ of $\boldsymbol{\delta}$ ) of the hypersurface defined by the graph $\boldsymbol{\delta} \mapsto \mathcal{L}_{\mathbf{W}}\left(\mathbf{w}_{\tau_{0}}^{\text {meas }}, \boldsymbol{\delta}\right)$ of the multidimensional log-likelihood function. For this given track stretch, the estimated optimal value $\boldsymbol{\delta}^{\text {opt }}$ is $\boldsymbol{\delta}^{\text {opt }}=(0.15,0.9,0.85,0.8)$. The adapted stochastic modeling of the track stretch is computed using Equations (15) and (16), such that

$$
\widetilde{\mathbf{X}}_{\tau}^{k}\left(\delta^{\mathrm{opt}, k}\right)=\left[Q^{k}\right]\left([\lambda]^{-1}[Q]^{T}\left(\mathbf{x}_{\tau}^{\text {meas }}\right)+\delta^{\mathrm{opt}, k} \mathbf{G}^{k}\right) \quad, \quad k=1,2,3,4
$$

For this track stretch, the stochastic response of the train dynamics is computed using the Monte-Carlo method with 2,000 realizations of the track geometry $\widetilde{\mathbf{X}}$. The 2,000 realizations of the track irregularities $\widetilde{\mathbf{X}}$ are generated using the local stochastic model of the track irregularities. Then, for each realization $\widetilde{\mathbf{X}}_{\tau}\left(\boldsymbol{\delta}^{\text {opt }} ; \theta^{\prime}\right)$ of the track geometry, the deterministic realization of the train response is computed with a multibody commercial software (Vampire). The static deterministic wheel-rail contact conditions are first computed by Vampire using the geometry of the wheel profile and of the rail profile. Then, the computations of the stochastic dynamic response of the train are performed in parallel, using a pre-processing 

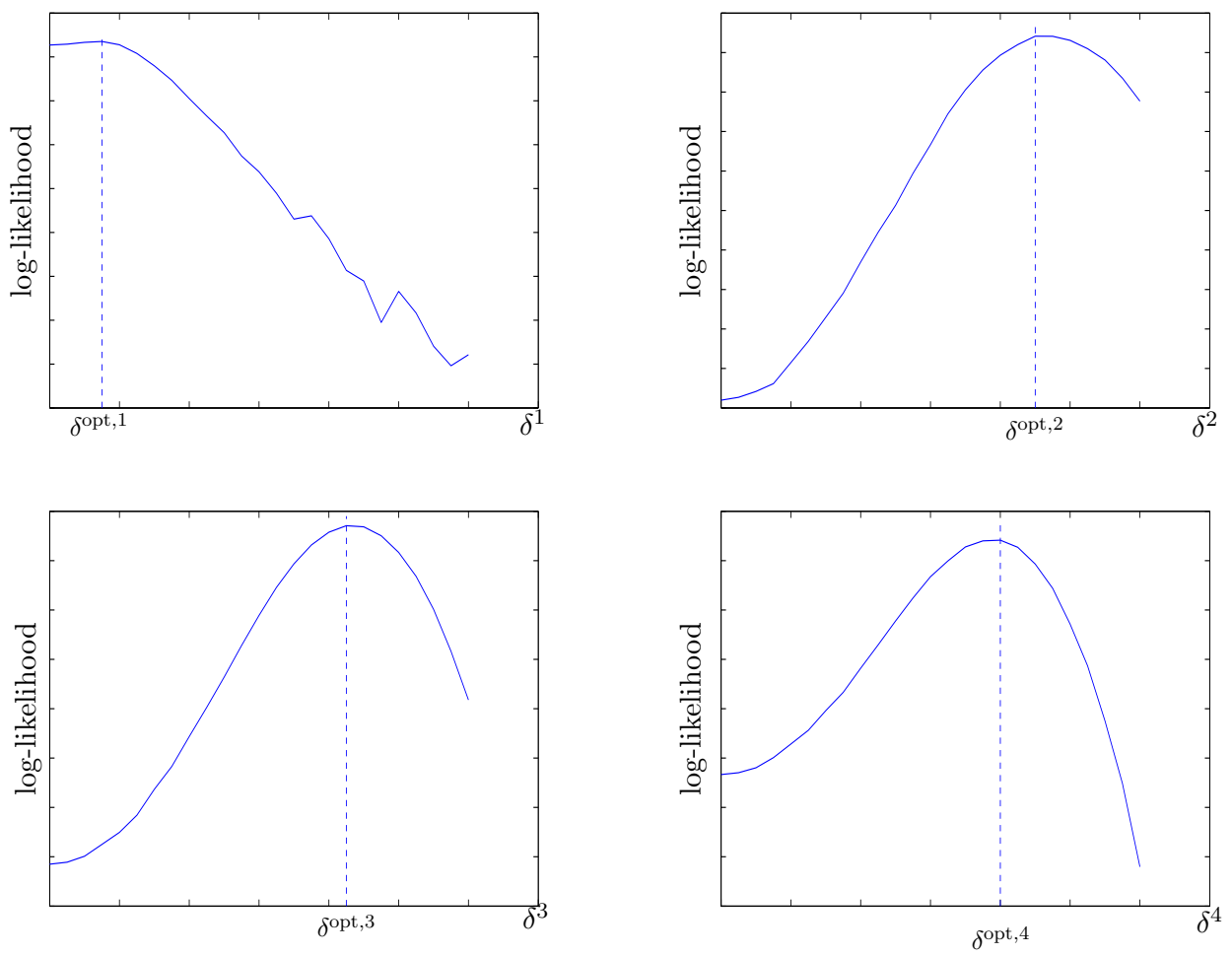

Figure 6. Sections of the log-likelihood $\mathcal{L}_{\mathbf{W}}\left(\mathbf{w}_{\tau_{0}}^{\text {meas }}, \boldsymbol{\delta}\right)$ for each component $\delta^{k}, k=1, \ldots, 4$, of $\boldsymbol{\delta}$

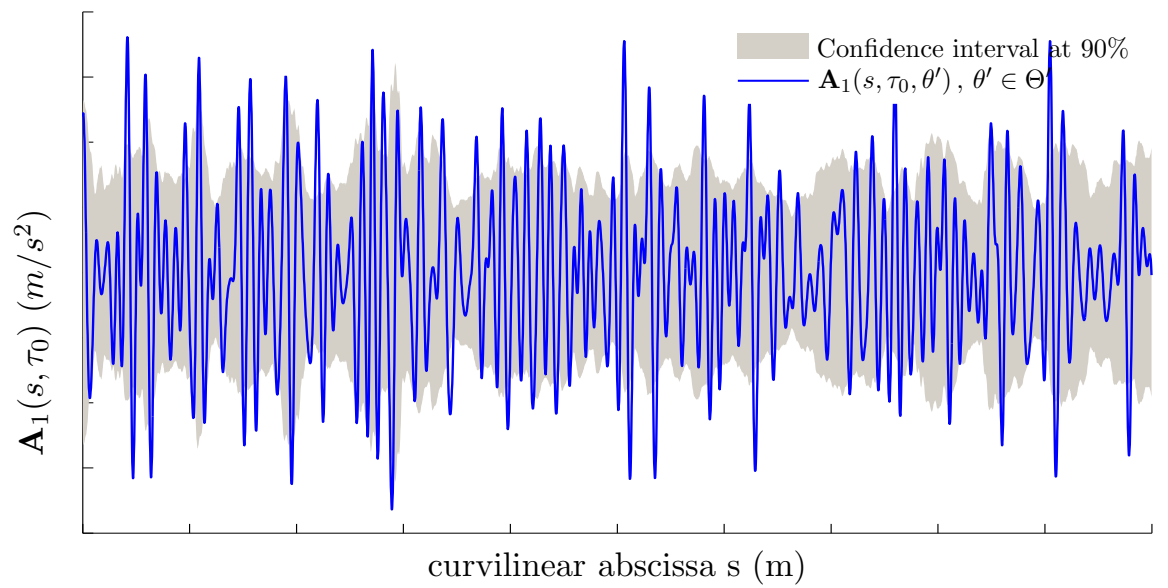

Figure 7. For a given track stretch, confidence region at $90 \%$ of the random lateral acceleration of the first bogie $s \mapsto \mathbf{A}_{1}\left(s, \tau_{0}\right)$ at $\tau=\tau_{0}$, and graph of one realization $s \mapsto \mathbf{A}_{1}\left(s, \tau_{0} ; \theta^{\prime}\right)$.

script developed to run Vampire in batch mode. For each run of Vampire, a realization of the stochastic track irregularities, the deterministic vehicle model, and the deterministic track model are used by Vampire. For the given track stretch and at $\tau=\tau_{0}$, the confidence region at $90 \%$ of the random lateral acceleration of the first bogie $s \mapsto \mathbf{A}_{1}\left(s, \tau_{0}\right)$ and the graph of one realization $s \mapsto \mathbf{A}_{1}\left(s, \tau_{0} ; \theta^{\prime}\right)$ are plotted in Figure 7. For confidentiality reasons, numerical values have been normalized. For the given track stretch, the simulated indicators vector $\mathbf{C}^{\text {sim }}$ is computed, as described in Section 4.1. Simultaneously, 2,000 independent realizations of noise $\mathbf{B}^{\text {out }}$ are generated with the polynomial chaos expansion identified in Section 4.3: An independent realization of noise $\mathbf{B}^{\text {out }}$ corresponds to each independent real- 
ization of random vector $\boldsymbol{\xi}$. Then, 2,000 independent realizations of the stochastic indicators vector $\mathbf{C}^{\text {mod }}$ are computed by combining the realizations of $\mathbf{C}^{\text {sim }}$ with the realizations of $\mathbf{B}^{\text {out }}$, as explained by Equation (20).

In order to analyse the results, the probability density functions of the components of $\mathbf{C}^{\text {mod }}$ and $\mathbf{C}^{\text {sim }}$ are estimated using the Gaussian kernel estimation method. For this track stretch, Figure 8 displays the probability density functions of the nine components of the random vectors $\mathbf{C}^{\text {mod }}$ and $\mathbf{C}^{\operatorname{sim}}$, at $\tau=\tau_{0}$ and at $\tau=\tau_{K}$.
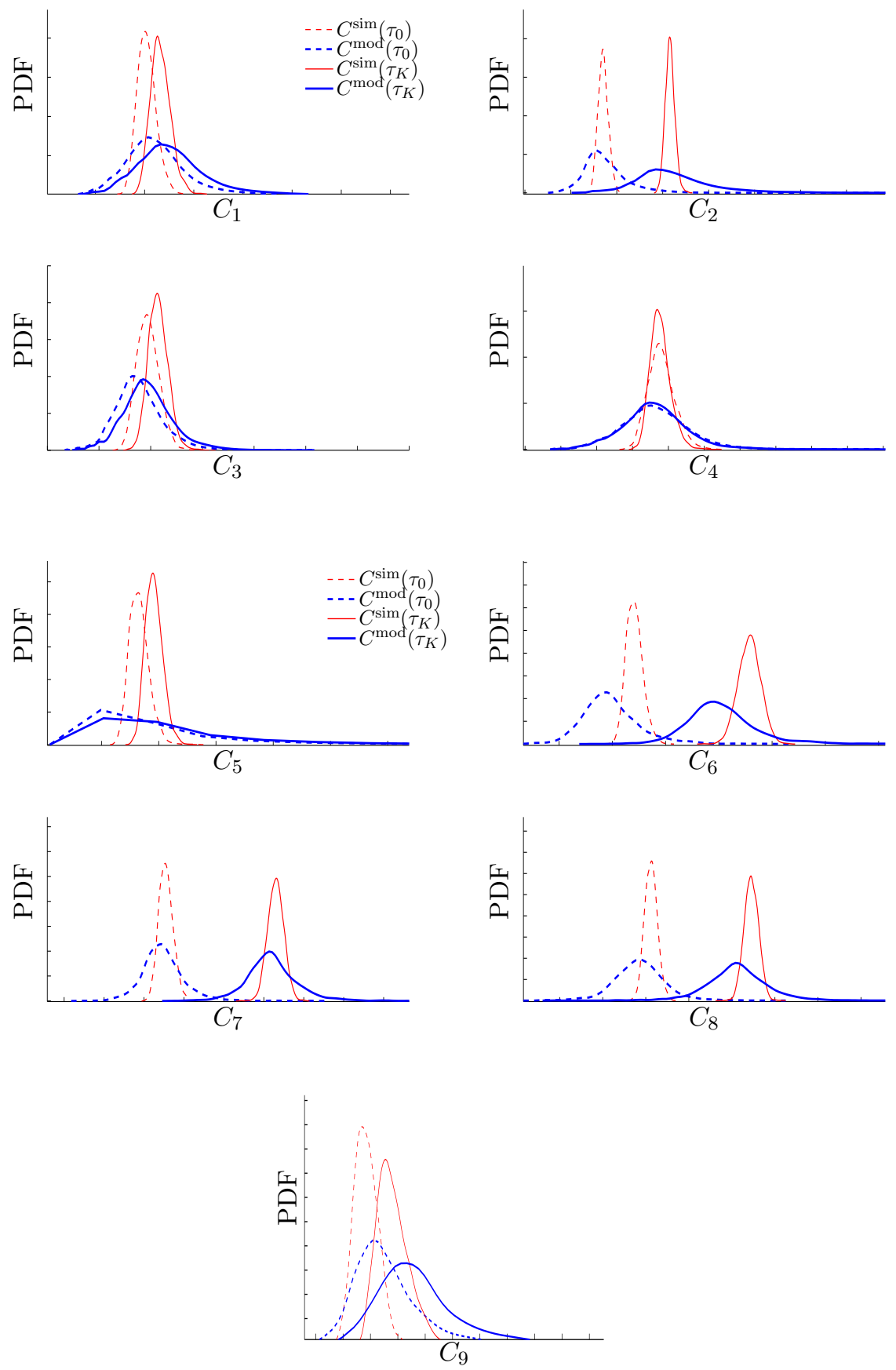

Figure 8. For the nine indicators, $j=1, \ldots, 9$, comparisons of the probability density functions of $C_{j}^{\text {sim }}\left(\tau_{0}\right)$ (red dashed line), $C_{j}^{\text {mod }}\left(\tau_{0}\right)$ (blue dashed line), $C_{j}^{\text {sim }}(K)$ (red solid line), $C_{j}^{\text {mod }}(K)$ (blue solid line). 
It can be seen on the figures that the components of $\mathbf{C}^{\text {mod }}$ are more dispersed than the components of $\mathbf{C}^{\text {sim }}$, because of the introduction of a model uncertainty in the train computational model. For each indicator, an evolution between $\tau_{0}$ and $\tau_{K}$ can be noticed. The distances between the probability density functions at $\tau_{0}$ and at $\tau_{K}$ are smaller for $\mathbf{C}^{\text {mod }}$ than for $\mathbf{C}^{\text {sim }}$.

\subsection{Long-term evolution of the dynamic response of the train}

For the given track stretch, for each component $j \in\{1, \ldots, 9\}$, and for given long time $\tau$, the probability density function (PDF) of the random variable $C_{j}^{\bmod }(\tau)$ is plotted in Figure 9. In Figure 9, the numerical values have been normalized by dividing the indicators values by a threshold value $\mathbf{c}^{*}=\left(c_{1}^{*}, c_{2}^{*}, c_{3}^{*}, c_{4}^{*}, c_{5}^{*}, c_{6}^{*}, c_{7}^{*}, c_{8}^{*}, c_{9}^{*}\right)$. The values of the components of $\mathbf{c}^{*}$ are chosen close to the limit values for the certification of the vehicles, that are given in [19]. The values given in [19] are arbitrarily adapted depending on the computed values of the indicators $\mathbf{C}^{\text {mod }}$. The dynamic indicators allow to assess the dynamic response of the train on a given track stretch and to observe its long-term evolution as a function of $\tau$. The long-term evolution of the PDFs of the random indicators shows a significant time evolution and an increase of the dispersion. Some indicators (for instance $C_{2}^{\bmod }$ ) have a bigger evolution than others.

An other way to analyse the long-term evolution of the indicators is proposed in Figure 10. On this Figure, for the long-term evolution of the random indicators $\tau \mapsto C_{2}^{\bmod }(\tau)$ and $\tau \mapsto C_{8}^{\bmod }(\tau)$, the mean functions and the confidence regions at $90 \%$ are plotted as a function of long time $\tau$. The successive values can be compared to the threshold values $c_{2}^{*}$ and $c_{8}^{*}$ displayed in the figure. Figures 9 and 10 show that, for every indicator, the mean value increases with long time $\tau$, excepted for $C_{4}^{\text {mod }}$, which is the lateral acceleration in the second car-body. It can be deduced that the comfort in the train is not deteriorated by the track irregularities for this track stretch due to the train suspensions, that operate as a filter. The indicators $C_{2}^{\text {mod }}, C_{6}^{\text {mod }}, C_{7}^{\text {mod }}$, and $C_{8}^{\text {mod }}$, which are related to the vertical direction, change much more than the indicators $C_{1}^{\mathrm{mod}}, C_{3}^{\mathrm{mod}}, C_{4}^{\mathrm{mod}}$, and $C_{5}^{\mathrm{mod}}$, which are related to the lateral direction. A detailed analysis of these results has been performed and has shown that this deterioration of the dynamic response of the train in the vertical direction is effectively due to a deterioration of the track stretch in the vertical direction. The roll of the vehicle (which is assessed by $C_{9}^{\text {mod}}$ ) changes much less than $C_{8}^{\text {mod }}$ that is also related to the dynamic response of the train in the vertical direction. These results show that all the indicators are not affected in the same way by the long-term evolution of the track irregularities. The dynamic response of the train cannot be directly deduced from the track irregularities because of the presence of nonlinearities in the train-track interaction.

In this paper, the methodology proposed allows for observing the influence of the long-term evolution of the track irregularities on the long-term evolution of the indicators related to the dynamic responses of the train, taking into account the dependence between the four track irregularities. The uncertainties in the computational model of the train dynamics are taken into account, which increases the robustness of the computational predictions of the statistics for the train indicators. It can then be concluded that the deterioration of the track irregularities does not have the same influence on all the indicators. For a given track stretch, the proposed methodology enables the railway network manager to start off the maintenance operations on the base of statistics related to the train indicators instead of experimental measurements of the track irregularities. The start off of the maintenance requires to set up a threshold on the indicators, as featured in 

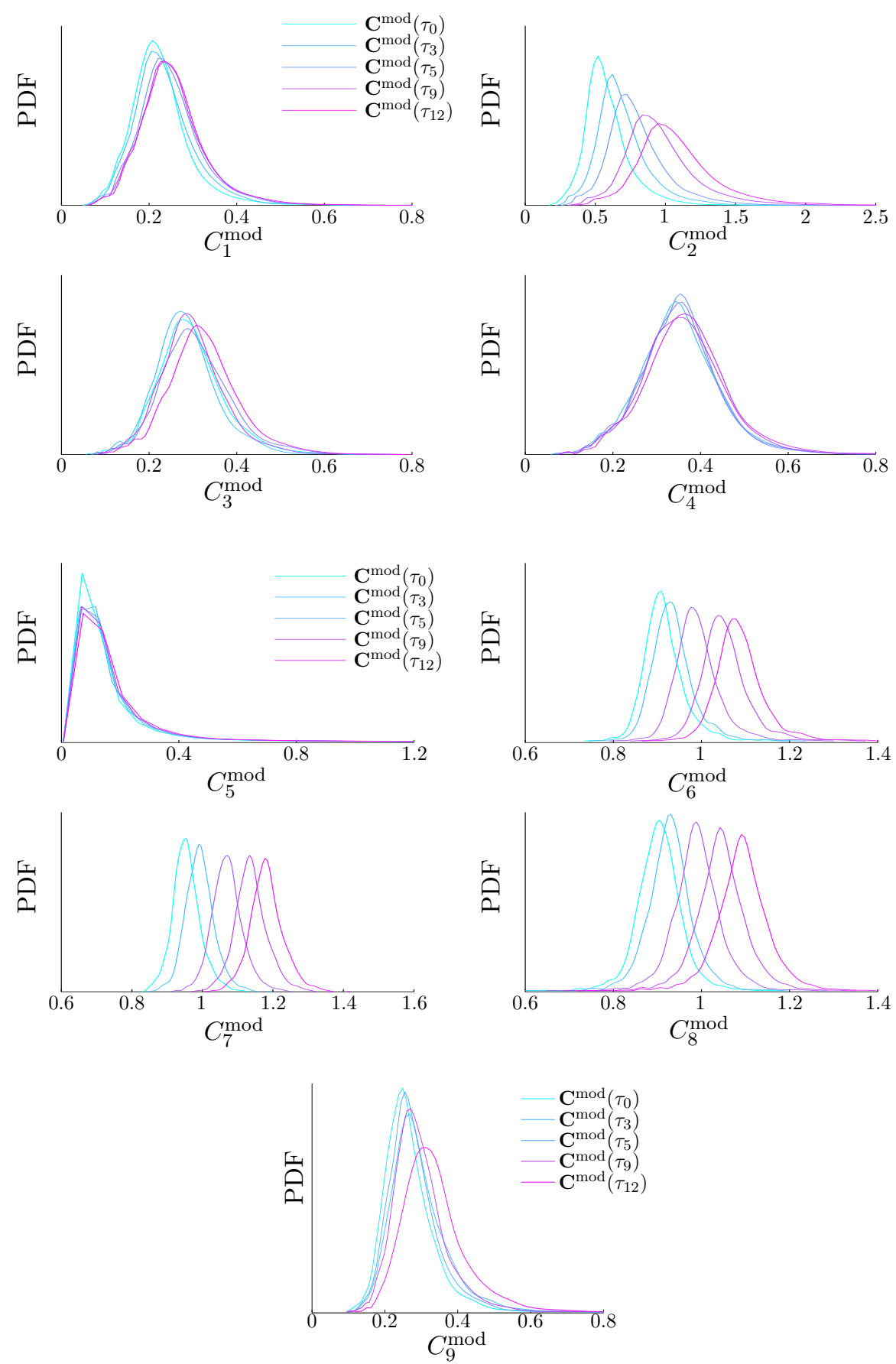

Figure 9. For each component $j \in\{1, \ldots, 9\}$ of the random indicators vector $\tau \mapsto \mathbf{C}^{\bmod }(\tau)$, graphs of the probability density function of $C_{j}^{\text {mod }}(\tau)$ for five values $\tau_{0}, \tau_{3}, \tau_{5}, \tau_{9}$, and $\tau_{12}$ of long time $\tau$.

Figure 10. The choice of the value for the threshold has to be done by the railway network manager according to its maintenance strategy.

\section{Conclusion}

In this work, indicators on the dynamic response of the train have been introduced, which allow to assess the long-term evolution of the train response on a given stretch of track, taking into account the variability of the track irregularities and the model 

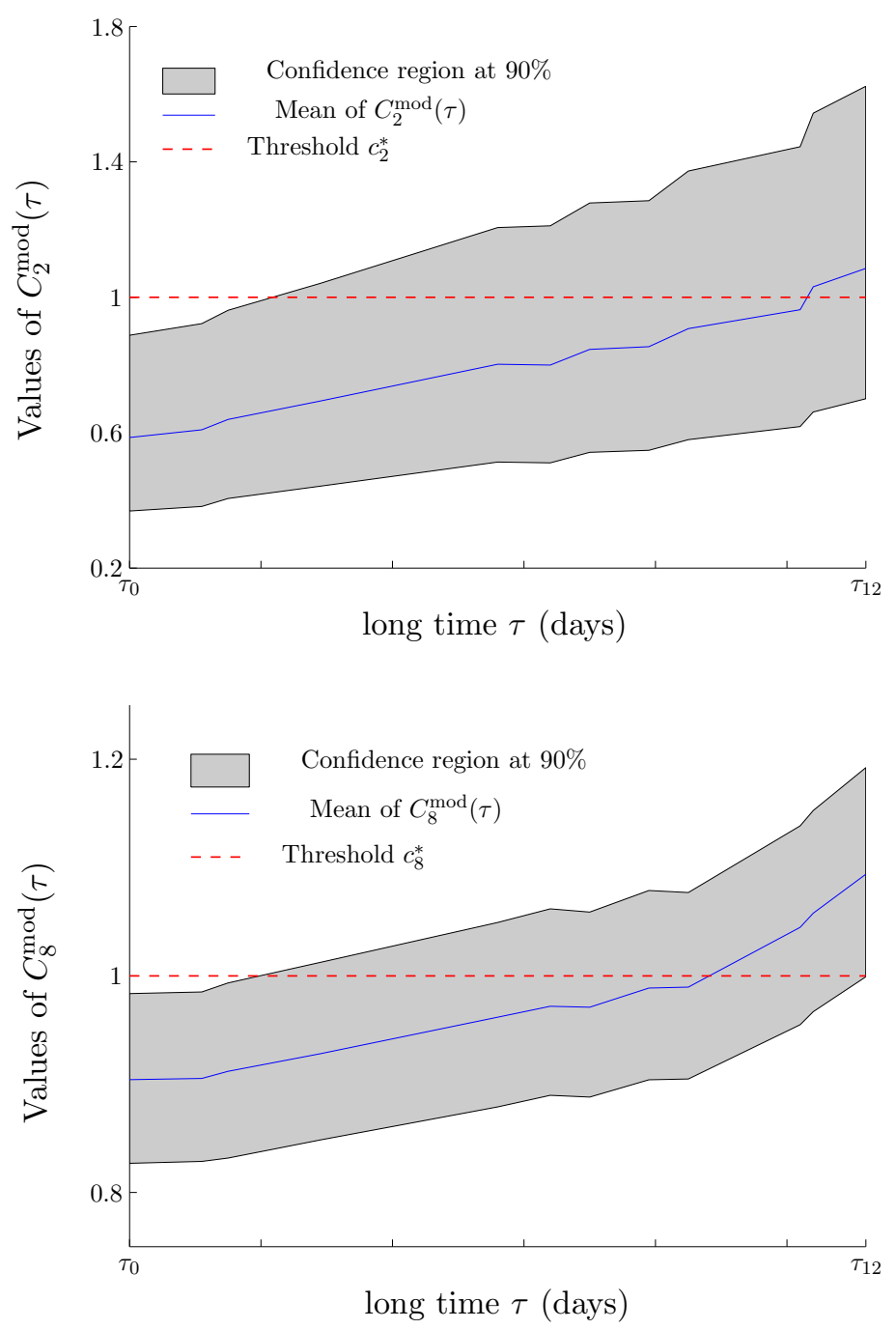

Figure 10. Long-term evolution of values of indicators: For components $j=2$ and $j=8$, mean function $\tau \mapsto \underline{C}_{j}(\tau)$ (blue solid line), confidence region of the random indicator $\tau \mapsto C_{j}^{\bmod }(\tau)$ at $90 \%$, and threshold level $c_{j}^{*}$.

uncertainties in the computational model of the train dynamics. Introducing model uncertainties increases the robustness of the computed indicators. It should be noticed that the dynamic indicators do not change in the same way, but in general each indicator has a mean value and a dispersion that increase as a function of the long time. As a prospect, the proposed stochastic model, which has been identified with experimental measurements, and which is experimentally validated, will be used for constructing a stochastic model for statistical prediction of long-term evolution of the indicators and thus, will allow for forecasting at what time a maintenance operation must be started off.

\section{Acknowledgments}

This study was supported by SNCF, the french railways company. 


\section{References}

[1] G. Lonnbark, Characterization of track irregularities with respect to vehicle response, Tech. Rep., TRITA AVE 2012:30, Aeronautical and Vehicle Engineering, Royal Institute of Technology, Stockholm, Sweden, 2012.

[2] G. Perrin, C. Soize, D. Duhamel, C. Funfschilling, Track irregularities stochastic modeling, Probabilistic Engineering Mechanics, Vol. 34 (2013), pp.123-130.

[3] N. Lestoille, C. Soize, G. Perrin, F. Funfschilling, Long Time Evolution of Train Dynamics with Respect to Track Irregularities, in Proceedings of The 2. International Congress on Railway Technology, Ajaccio, France, 8-11 April 2014, Civil-Comp Press, Lisbon, 2014.

[4] G. Perrin, Random fields and associated statistical inverse problems for uncertainty quantifications - Applications to railway track geometries for high-speed trains dynamical responses and risk assessment, Ph. D. Diss., Université Paris-Est, France, (2013).

[5] G. Perrin, C. Soize, D. Duhamel, C. Funfschilling, Quantification of the influence of the track geometry variability on the train dynamics, Mechanical System and Signal processing, Accepted for publication 05 January 2015.

[6] G. Foeillet, F. Coudert, V. Delcourt, IRIS 320 is a global concept inspection vehicle merging engineering and R\&SD tools for infrastructure maintenance, in Proceedings of the Eight World Congress on Railway Research, Seoul, South Korea, 2008.

[7] F. Coudert, B. Richard, IRIS 320 GEOV: a new track geometry recoding system designed continuing on from the Mauzin track recording coaches, Revue Générale des Chemins de Fer, JUN 2009, pp. $7-22$.

[8] H.B. Zheng, Q.S. Yan, J.L. Hu, and Z. Chen, Numerical simulation of railway track irregularities based on stochastic expansion method of standard orthogonal basis, Applied Mechanics and Materials, Vol. 178 (2012), pp.1373-1378.

[9] R.N. Iyengar, O.R. Jaiswal, A new model for non-Gaussian random excitations, Probabilistic Engineering Mechanics, Vol. 8 (1993), pp.281-287.

[10] X. Lei and N.A. Noda, Analyses of dynamic response of vehicle and track coupling with random irregularity of track vertical profile, Journal of Sound and Vibration, Vol. 258(1) (2002), pp.147-165.

[11] A. Hamid and A. Gross, Track-quality indices and track-degradation models for maintenance-of-way planning, Transportation Research Record: Journal of the Transport Research Board, Vol. 802 (1981), pp. $2-8$.

[12] G. Perrin, C. Soize, D. Duhamel, and C. Funfschilling, Modeling the track geometry variability, In 10th World Congress on Computational Mechanics, Sao Paulo, Brazil, 2012.

13] G. Perrin, C. Soize, D. Duhamel, C. Funfschilling, Identification of polynomial chaos representations in high dimension from a set of realizations, SIAM Journal of Scientific Computing, Vol. 34, No. 6 (2012), pp. A2917-A2945.

[14] C.Soize, Identification of high-dimension polynomial chaos expansions with random coefficients for non-Gaussian tensor-valued random fields using partial and limited experimental data, Computer Methods in Applied Mechanics and Engineering, Vol. 199 (2010), pp. 2150-2164.

[15] G. Perrin, C. Soize, D. Duhamel, C. Funfschilling, Karhunen-Love expansion revisited for vectorvalued random fields: scaling, errors and optimal basis, Journal of Computational Physics, Vol. 242, No. 1 (2013), pp. 607-622.

[16] G. Perrin, C. Soize, D. Duhamel, C. Funfschilling, A posteriori error and optimal reduced basis for stochastic processes defined by a finite set of realizations, SIAM/ASA, Journal of Uncertainty Quantification, Vol. 2, (2014), pp. 745-762.

[17] G. R. Terrell, D. W. Scott, Variable Kernel Density Estimation, The Annnals of Statistics, Vol. 20, No. 3 (1992), pp. 1236-1265.

[18] A. W. Bowman and W. Azzalini, Applied Smoothing Techniques for Data Analysis, Oxford University Press, Oxford, 1997.

[19] UIC, Testing and approval of railway vehicles from the point of view of their dynamic behaviour Safety - Track fatigue - Running behaviour UIC Leaflet 518, Paris, France, 2009

[20] S. Kraft, Parameter identification for a TGV model, Ph. D. diss., Ecole Centrale Paris, France, 2012.

[21] C. Soize, Stochastic Models of Uncertainties in Computational Mechanics, ASCE, Reston, VA, USA, 2012.

[22] R. Ghanem, P. Spanos, Stochastic finite elements: a spectral approach, Dover Publications, New York, 2003 (1st edition: Springer-Verlag, New York, 1991).

[23] O. Le Maitre, O. Knio, Spectral Methods for Uncertainty Quantification, Springer, 2010. 